\title{
Identificação de danos estruturais utilizando dados no domínio do tempo e critério D-ótimo
}

\author{
Luciano S. Rangel, Leonardo T. Stutz, Isabela C. S. S. Rangel, \\ Universidade do Estado do Rio de Janeiro, Instituto Politécnico, \\ 28.610-974, Nova Friburgo, RJ, \\ E-mail: lrangel@iprj.uerj.br, ltstutz@iprj.uerj.br, isilva@iprj.uerj.br.
}

\begin{abstract}
Resumo: O presente trabalho aborda o problema de identificação de danos em uma estrutura a partir de sua resposta impulsiva. No modelo adotado, a integridade estrutural é continuamente descrita por um parâmetro nodal de coesão. Sendo assim, o Modelo de Elementos Finitos (MEF) é utilizado para discretizar tanto o campo de deslocamentos, quanto o campo de coesão. O problema de identificação de danos é, então, definido como um problema de otimização, cujo objetivo é minimizar, em relação a um vetor de parâmetros nodais de coesão, um funcional definido a partir da diferença entre a resposta impulsiva experimental e a correspondente resposta prevista por um MEF da estrutura. A identificação de danos estruturais baseadas no domínio do tempo apresenta como vantagens a aplicabilidade em sistemas lineares e/ou com elevados níveis de amortecimento, além de apresentar uma elevada sensibilidade à presença de pequenos danos. Estudos numéricos foram realizados considerando-se um modelo de viga de Euler-Bernoulli simplesmente apoiada. Para a determinação do posicionamento ótimo do sensor de deslocamento, a ser utilizado no processo de identificação de danos, foi considerado o critério D-ótimo. Para a resolução do problema inverso de identificação de danos foi considerado o método de otimização Evolução Diferencial (ED). Simulações numéricas, considerando dados corrompidos com ruído aditivo, foram realizadas com o intuito de avaliar a potencialidade da metodologia de identificação de danos, assim como a influência da posição do sensor no processo de identificação. Com os resultados obtidos, percebe-se que o critério D-ótimo é de fundamental importância para a identificação de danos.
\end{abstract}

Palavras-chave: Identificação de danos estruturais. Modelo de Elementos Finitos. Modelo de dano contínuo. Resposta impulsiva. Critério D-ótimo.

\section{Introdução}

Estruturas de engenharia estão expostas a processos de deterioração e à ocorrência de dano durante sua vida útil. Juntamente com o envelhecimento e desgastes intrínsecos à idade, eventos distintos podem contribuir para tais acontecimentos, como fatores naturais e outros provocados pelo homem.

O desenvolvimento de procedimentos de identificação de danos é indispensável para garantir a segurança e aumentar a vida útil da estrutura. Nos últimos anos, técnicas não destrutivas de identificação de danos estruturais, fundamentadas na resposta dinâmica da estrutura, vem se tornando uma prática utilizada nas indústrias da construção civil, mecânica e aeroespacial [1]. O contínuo monitoramento da estrutura e a identificação de danos num estágio inicial contribuem para a redução dos custos de manutenção e reparo, além de aumentar sua confiabilidade e vida útil.

As técnicas de identificação de danos fundamentados na resposta dinâmica da estrutura são, de forma geral, classificados em três tipos, de acordo com o domínio dos dados utilizados: técnicas no domínio do tempo [2], técnicas no domínio da frequência [3] e técnicas modais [4]. $\mathrm{O}$ 
presente trabalho considera a identificação de danos a partir da resposta impulsiva da estrutura, sendo portanto, uma técnica fundamentada no domínio do tempo.

Neste trabalho, estuda-se o problema de identificação de danos estruturais em uma viga de Euler-Bernoulli simplesmente apoiada. A resposta impulsiva da estrutura foi considerada na formulação do problema inverso. O trabalho é organizado como se segue. Na formulação do problema direto apresenta-se a definição do parâmetro de coesão $\beta$, utilizado para descrever o estado de dano na estrutura [5], e o modelo matemático do problema direto de vibrações de uma viga de Euler-Bernoulli no domínio do tempo, via MEF. Na formulação do problema inverso é apresentada, de forma sucinta, o problema de identificação de danos a ser resolvido. Nos resultados numéricos é apresentada a estratégia utilizada para a escolha do posicionamento ótimo do sensor de deslocamento e os resultados referentes a estimação dos parâmetros nodais de coesão de uma viga de Euler-Bernoulli simplesmente apoiada. São considerados dados experimentais sintéticos corrompidos com ruído aditivo. Foi utilizado o método de otimização ED, com o propósito de solucionar o problema inverso de identificação de danos e foi utilizado o software MATLAB para a implementação das simulações numéricas. Por último, são apresentadas as conclusões obtidas com a presente pesquisa e são realizadas algumas sugestões para trabalhos futuros.

\section{Formulação do Problema Direto}

Na estratégia de identificação de danos adotada, a integridade da estrutura é considerada como sendo continuamente descrita, no domínio do corpo, por um parâmetro estrutural denominado parâmetro nodal de coesão $(\beta)$ [5]. Este parâmetro está relacionado com a ligação entre os pontos materiais e pode ser interpretado como uma medida do estado de coesão local do material, onde $0 \leq \beta \leq 1$. Se $\beta=1$, considera-se que todas as ligações entre os pontos materiais foram preservadas, ou seja, não há defeito na estrutura. Se $\beta=0$, considera-se uma ruptura local, pois todas as ligações entre os pontos materiais foram desfeitas.

Neste trabalho, considerou-se que o dano afeta apenas as propriedades elásticas da estrutura, hipótese comumente adotada na literatura. Deste modo, a matriz de rigidez do MEF da estrutura pode ser escrita como

$$
\mathbf{K}\left(\beta_{h}\right)=\int_{\Omega} \beta_{h}(x) E_{0} I_{0} \mathbf{H}^{T}(x) \mathbf{H}(x) d \Omega,
$$

onde $\mathbf{H}$ é o operador diferencial discretizado, $E_{0}$ e $I_{0}$ são, respectivamente, os valores nominais do módulo de elasticidade e do momento de inércia de área e $\beta$ representa o campo de coesão no domínio elástico $\Omega$ da estrutura. Deve-se enfatizar que a discretização do campo de coesão $\beta$ não depende da discretização do campo de deslocamentos, de forma que diferentes malhas podem ser adotadas.

A partir da Eq. (1), tem-se que a rigidez à flexão ao longo da viga é dada por

$$
E(x) I(x)=\beta(x) E_{0} I_{0} .
$$

Portanto, o parâmetro de coesão representa qualquer alteração, provocada pela presença de danos estruturais, na rigidez à flexão da estrutura. Por simplicidade, considerando-se uma viga de seção transversal retangular e com módulo de elasticidade uniforme, o campo de coesão pode ser escrito como

$$
\beta(x)=\left(\frac{h(x)}{h_{0}}\right)^{3}
$$

onde $h_{0}$ e $h(x)$, indicam, respectivamente, a espessura nominal e a espessura da viga na posição $x$.

A equação de movimento do sistema, após a discretização espacial a partir do MEF, é dada por

$$
\mathbf{M} \ddot{\mathbf{q}}+\mathbf{D} \dot{\mathbf{q}}+\mathbf{K}(\boldsymbol{\beta}) \mathbf{q}=\mathbf{f},
$$


onde $\mathbf{q}$ representa o vetor de coordenadas generalizadas, $\mathbf{M}$ é a matriz de massa, $\mathbf{D}$ é a matriz de amortecimento, $\mathbf{K}(\boldsymbol{\beta})$ é a matriz de rigidez, parametrizada pelo parâmetro de coesão, e f é o vetor de carregamentos generalizados. O vetor de parâmetros nodais de coesão é definido como

$$
\boldsymbol{\beta}=\left[\beta_{1}, \beta_{2}, \ldots, \beta_{n p}\right]^{T},
$$

onde $n p$ é o número total de parâmetros de coesão do modelo. Portanto, considerando-se as Eqs. (3) e (5), nos nós defeituosos tem-se $h(x) / h_{0}<1$, e nos nós onde não há danos, tem-se $h(x) / h_{0}=1$.

\section{Formulação do Problema Inverso}

Para a formulação do problema de identificação de danos no domínio do tempo, necessariamente, define-se o vetor de resposta generalizada como se segue. Seja $\mathbf{y}_{E i}$ a resposta da estrutura medida no sensor $i$, formulada como

$$
\mathbf{y}_{E i}=\left[\begin{array}{llll}
y_{E i}\left(t_{1}\right) & y_{E i}\left(t_{2}\right) & \ldots & y_{E i}\left(t_{N}\right)
\end{array}\right]^{T},
$$

onde $y_{E i}\left(t_{j}\right)$ representa a resposta amostrada, no sensor $i$, no instante de tempo $t_{j}$ e $N$ é o número de amostras no tempo consideradas no processo de identificação. Descreve-se o vetor de resposta generalizada como

$$
\mathbf{z}_{E}=\left[\begin{array}{llll}
\mathbf{y}_{E 1} & \mathbf{y}_{E 2} & \cdots & \mathbf{y}_{E m}
\end{array}\right]^{T},
$$

sendo $m$ o número de sensores utilizados no ensaio experimental.

Define-se o problema inverso de identificação de danos como o seguinte problema de minimização

$$
\min _{\boldsymbol{\beta}} \frac{1}{2}\left\|\mathbf{z}_{E}-\mathbf{z}(\boldsymbol{\beta})\right\|^{2}
$$

onde $\mathbf{z}(\boldsymbol{\beta})$ corresponde ao vetor de resposta generalizada previsto pelo modelo da estrutura.

Pode-se obter a resposta estrutural em questão a partir de sensores comumente utilizados em ensaios de vibrações, como os acelerômetros e sensores de deslocamento, por exemplo, e para que esta contenha informações a respeito do comportamento da estrutura em alta frequência, excitações de impacto ou aleatórias são consideradas. No presente trabalho, para a resolução do problema inverso em questão, utiliza-se o método Evolução Diferencial [6].

\section{Resultados Numéricos}

Nos resultados que se seguem, considerou-se uma viga de aço, simplesmente apoiada, de Euler-Bernoulli, com os parâmetros geométricos e materiais descritos na Tabela 1.

\begin{tabular}{cc}
\hline \hline Comprimento & $1,46 \mathrm{~m}$ \\
Espessura & $7,9375 \times 10^{-3} \mathrm{~m}$ \\
Largura & $7,62 \times 10^{-2} \mathrm{~m}$ \\
Momento de Inércia de Área & $3,1756 \times 10^{-9} \mathrm{~m}^{4}$ \\
Módulo de Elaticidade & $2,07 \times 10^{11} \mathrm{~Pa}$ \\
Massa específica & $7,85 \times 10^{3} \mathrm{~kg} / \mathrm{m}^{3}$ \\
\hline \hline
\end{tabular}

Tabela 1: Propriedades nominais da viga.

A viga foi discretizada em 24 elementos, onde cada elemento apresenta dois nós e cada nó, dois graus de liberdade (GDL) - um de rotação e outro de translação - e um parâmetro nodal de coesão. Portanto, a estrutura possui 25 parâmetros nodais de coesão e, devido às condições de contorno, 48 GDL. 
A resposta impulsiva da viga foi simulada considerando-se uma excitação de impacto a $0,365 \mathrm{~m}$ da extremidade esquerda da mesma. Simulou-se $10 \mathrm{~s}$ de resposta de deslocamento em intervalos de tempo constantes de 0,001 s, totalizando assim, 10000 amostras no tempo.

Para a determinação do posicionamento ótimo do sensor de deslocamento e do número de pontos da resposta impulsiva que serão utilizados no processo de identificação de danos, considerou-se o critério D-ótimo [7], que tem como objetivo maximizar o determinante da matriz de informação $\left(\mathbf{X}^{T} \mathbf{X}\right)$, onde $\mathbf{X}$ é a matriz de sensibilidade, definida como

$$
\mathbf{X}=\left[\frac{\partial \mathbf{z}}{\partial \boldsymbol{\beta}}\right] .
$$

Para o cálculo da matriz de sensibilidade, a reposta impulsiva da viga foi determinada para três cenários de danos distintos, definidos a partir de uma redução de $10 \%$ na espessura relativa da viga, separadamente, nas posições 0,1217, 0,7908 e 1,3383 m.

A Tabela 2 apresenta as diferentes posições em metros (em relação à extremidade esquerda da viga) para a possível fixação do sensor na viga. A Tabela 3, por sua vez, apresenta os números de pontos da resposta impulsiva, que serão considerados para o cálculo da matriz de sensibilidade.

\begin{tabular}{ccccccc}
\hline \hline & $(\mathrm{S} 1)$ & $(\mathrm{S} 2)$ & $(\mathrm{S} 3)$ & $(\mathrm{S} 4)$ & $(\mathrm{S} 5)$ & $(\mathrm{S} 6)$ \\
\hline Sensor $(\mathrm{m})$ & 0,2433 & 0,3650 & 0,4867 & 0,7300 & 0,9733 & 1,2167 \\
\hline \hline
\end{tabular}

Tabela 2: Posições do sensor ao longo da viga.

\begin{tabular}{cccccc}
\hline \hline & $\mathrm{N} 1$ & $\mathrm{~N} 2$ & $\mathrm{~N} 3$ & $\mathrm{~N} 4$ & $\mathrm{~N} 5$ \\
\hline Número de pontos & 125 & 250 & 500 & 1000 & 5000 \\
\hline \hline
\end{tabular}

Tabela 3: Número de pontos da resposta impulsiva

A Figura 1 apresenta o determinate da matriz de informação, calculado para as diferentes posições do sensor em função do número de pontos, para os diferentes cenários de dano.

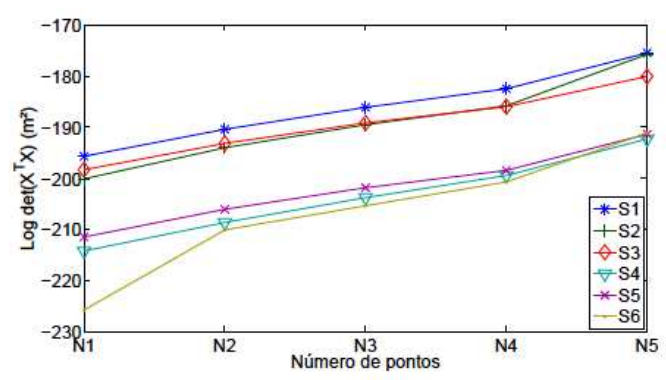

(a) $h(0,1217)=0,9 h_{0}$

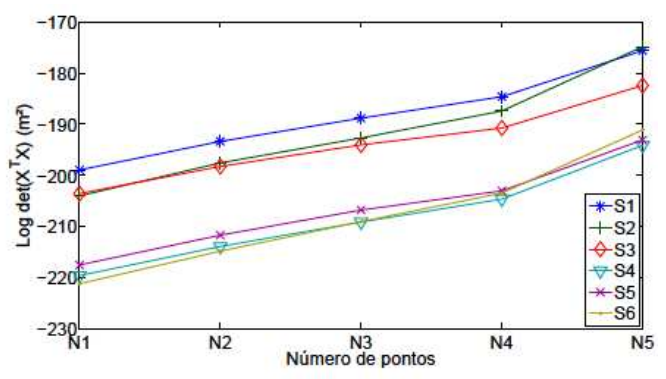

(b) $h(0,7908)=0,9 h_{0}$

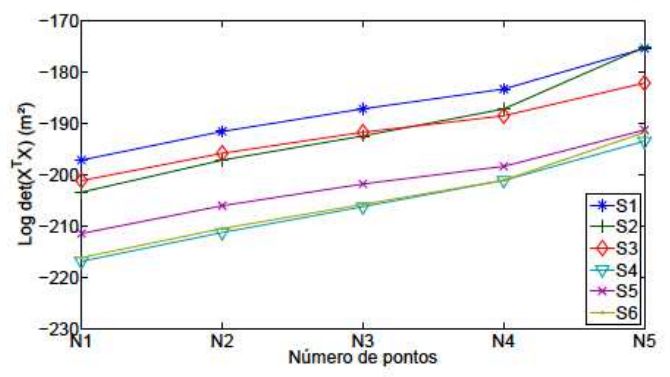

(c) $h(1,3383)=0,9 h_{0}$

Figura 1: Determinante da matriz $\left(\mathbf{X}^{T} \mathbf{X}\right)$ calculada para diferentes sensores e cenários de dano 
Considerando-se a Figura 1, pode-se determinar que em 86,67\% dos casos, os maiores valores do determinante da matriz $\left(\mathbf{X}^{T} \mathbf{X}\right)$ foram calculados com o sensor S1 e os menores valores foram calculados com os sensores S4 (60\% das simulações) e S6 (40\% das simulações). Sendo assim, assume-se que a posição ótima para a fixação do sensor na estrutura é a 0, $2433 \mathrm{~m}$ da extremidade esquerda da viga, ou seja, a posição do sensor S1.

Nos resultados que se seguem, considerou-se o cenário de dano composto por duas regiões danificadas, definidas por $h(x) / h_{0}=0,9$ (ou seja $\beta(x)=0,7290$ ), nas posições $x=0,5475 \mathrm{e}$ $1,2775 \mathrm{~m}$. Adotou-se a mesma malha de elementos para aproximar o campo de deslocamentos e o campo de coesão (dano). O vetor de resposta generalizada da estrutura, obtido a partir do MEF com os valores prescritos dos parâmetros nodais de coesão, representam os dados experimentais sintéticos utilizados no processo de identificação de danos. A esses dados, considerou-se a adição de ruído de distribuição gaussiana, de média nula e desvio padrão determinado indiretamente pela razão-sinal-ruído (SNR), definida como

$$
\mathrm{SNR}=10 \log \left(P_{s} / P_{r}\right),
$$

onde $P_{s}$ e $P_{r}$ são, respectivamente, a potência do sinal e do ruído.

Os casos considerados são apresentados na Tabela 4.

\begin{tabular}{cccc}
\hline \hline Caso & SNR $(\mathrm{dB})$ & Sensor utilizado & Número de pontos \\
\hline 1 & 20 & $\mathrm{~S} 1$ & $\mathrm{~N} 1$ \\
2 & 20 & $\mathrm{~S} 4$ & $\mathrm{~N} 1$ \\
\hline \hline
\end{tabular}

Tabela 4: Casos de estudo

No Caso 1, considerou-se os 125 primeiros pontos da resposta impulsiva da estrutura, medida no no sensor de deslocamento S1 - que possui posicionamento ótimo, segundo o critério Dótimo -; além disso, considerou-se a resposta impulsiva corrompida com um ruído aditivo com $\mathrm{SNR}=20 \mathrm{~dB}$. No Caso 2, considerou-se os 125 primeiros pontos da resposta impulsiva da estrutura, medida no no sensor de deslocamento S4 - que representa o sensor que produziu os menores valores para o determinante da matriz de informação - e a mesma intensidade de ruído adicionado aos dados experimentais.

A seguir, apresenta-se os resultados da estimação dos parâmetros nodais de coesão. Devido à aleatoriedade dos métodos estocásticos, realiza-se 10 simulações com o método ED, todas utilizando a mesma resposta impulsiva da estrutura danificada.

A Figura 2, apresenta os resultados da identificação de danos obtidos de 10 simulações com o método ED para o Caso 1.

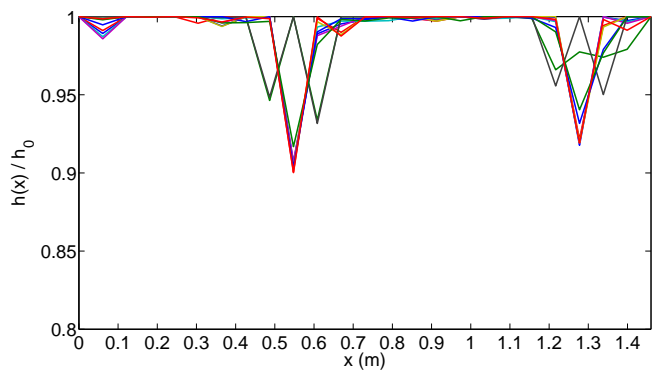

Figura 2: Identificação de danos das 10 simulações com o método ED para o Caso 1.

Da Figura 2, pode-se notar que, embora alguns resultados indicaram a presença de dano em regiões intactas da estrutura, em geral, tem-se uma boa estimativa da atual região danificada.

A Figura 3, apresenta a média dos 10 resultados de identificação obtidos com o método ED. 


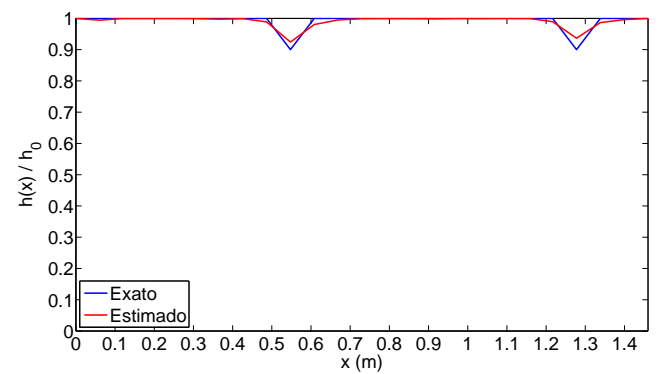

Figura 3: Identificação de danos obtida da média dos 10 resultados individuais do método ED para o Caso 1.

Como pode ser observado na Figura 3, considerando-se a média dos resultados individuais, a abordagem proposta foi capaz de identificar com acurácia a localização do dano, porém indicando uma intensidade ligeiramente menor nas regiões afetadas.

A Figura 4 apresenta os resultados individuais obtidos de 10 simulações com o método ED para o Caso 2.

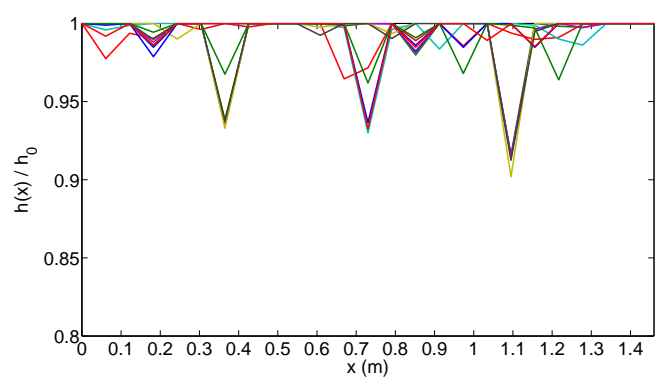

Figura 4: Identificação de danos das 10 simulações com o método ED para o Caso 2.

Na Figura 4, pode-se observar que alguns dos resultados fornecidos pelo método ED indicam a presença de danos, com elevada intensidade, em regiões intactas da estrutura.

A Figura 5, apresenta a média dos 10 resultados de identificação obtidos com o método ED.

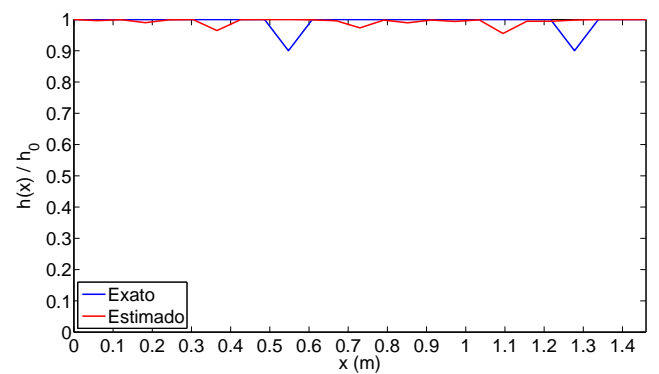

Figura 5: Identificação de danos obtida da média dos 10 resultados individuais do método ED para o Caso 2.

A Figura 5, apresenta o campo de dano exato e estimado. Conclui-se que, o campo de dano estimado, não recupera de forma correta a localização e intensidade dos danos, apresentando falsas regiões de danos.

Portanto, pode-se concluir que o posicionamento do sensor interferiu de forma significativa no resultado de identificação de danos apresentado pelo método ED.

\section{Conclusões}


No presente trabalho, o campo de dano da estrura foi descrito de forma contínua por um parâmetro estrutural denominado parâmetro de coesão. A discretização espacial do campo de coesão foi obtida através do MEF. O problema de identificação de danos foi definido como um problema de minimização, onde o objetivo é minimizar, em relação ao parâmetro nodal de coesão, um funcional definido a partir da diferença entre a resposta impulsiva prevista por um MEF da estrutura e a correspondente resposta obtida experimentalmente. O critério Dótimo foi utilizado para selecionar a posição ótima, dentre algumas posições pré-definidas, do sensor de deslocamento utilizado para a medição da resposta impulsiva. O método de otimização estocástico Evolução Diferencial foi considerado para resolver o problema inverso de identificação de danos. Das análises numéricas apresentadas, observa-se claramente que posição do sensor influenciou de forma significativa nos resultados de identificação. Quando a posição ótima do sensor foi considerada no processo de identificação, a abordagem proposta foi capaz de identificar com acurária o campo de dano da estrutura, mesmo na presença de um elevado nível de ruído nos dados experimentais sintéticos. O mesmo não ocorreu quando a posição do sensor foi alterada para aquela que apresentou um menor desempenho, segundo o critério D-ótimo. Nesse caso, falsas indicações de dano foram obtidas.

Sugestões para trabalhos futuros é a análise de técnicas de localização de danos com o intuito de minimizar o número de parâmetros nodais de coesão a serem atualizados e utilizar dados no domínio do tempo, juntamente com o critério D-ótimo, para problemas de identificação de danos em placas.

\section{Referências}

[1] J.V.A. Santos, N.M.M. Maia, C.M.M. Soares, Structural damage identification: A survey. In: Topping BHV, Papadrakakis M (Ed.), Trends in computational structures technology, Stirlingshire, Scotland, 2008.

[2] P. Cacciola, N. Maugeri, G. Muscolino, Structural identification through the measure of deterministic and stochastic time-domain dynamic response. Computers and Structures, 89 (2011) 1812-1819.

[3] J.V.A. Santos, C.M.M. Soares, C.A.M. Soares, N.M.M. Maia, Structural damage identification in laminated structures using FRF data. Composite Structures, 67 (2005) 239-249.

[4] A. Tomaszewska, Influence of statistical errors on damage detection based on structural flexibility and mode shape curvature. Computers and Structures, 88 (2010) 154-164.

[5] L. T. Stutz, D. A. Castello, F. A. Rochinha, A flexibility-based continuum damage identification approach. Journal of Sound and Vibration, 279, pp. 641-667, 2005.

[6] R. Storn, K. Price, Differential Evolution - A simple and efficient adaptive scheme for global optimization over continuous spaces. Journal of Global Optimization Vol. 11, pp. 341-359, Berkekey, 1997.

[7] P. F. Aguiar, B. Bourguignon, M. S. Khouts, D. L. Massart, Tutorial: D-optimal designs. Chemomrtrics and Intelligent Laboratory Systems. Vol.30. pp. 199-210, 1995. 